\title{
Modeling Precipitate Dissolution in Hardened Aluminium Alloys using Neural Networks
}

\author{
R. Lopez ${ }^{1}$, B. Ducoeur ${ }^{2}$, M. Chiumenti ${ }^{1}$, B. de Meester ${ }^{2}$, C. Agelet de Saracibar $^{1}$
}

\author{
${ }^{1}$ International Center for Numerical Methods in Engineering (CIMNE), Barcelona, Spain \\ URL: www.cimne.upc.edu, E-mail: rlopez@cimne.upc.edu, chiument@cimne.upc.eud, \\ agelet@cimne.upc.edu \\ ${ }^{2}$ Université catholique de Louvain-la-Neuve, Louvain-la-Neuve, Belgium \\ URL: http://www.uclouvain.be, E-mail: benjamin.ducoeur@uclouvain.be, \\ bruno.demeester@uclouvain.be
}

ABSTRACT: This work presents a neural networks approach for finding the effective activation energy and modeling the dissolution rate of hardening precipitates in aluminium alloys using inverse analysis. As way of illustration, a class of multilayer perceptron extended with independent parameters is applied for that purpose to aluminium alloys AA-7449-T79, AA-2198-T8 and AA-6005A-T6.

KEYWORDS: Neural networks, Multilayer perceptron, Inverse analysis, Microstructural modeling

\section{INTRODUCTION}

In heat-treatable aluminium alloys, the dominant microstructural effects are due to changes in the precipitation state of the material, which provide the principal strengthening mechanism. Modeling the dissolution rate of hardening precipitates is therefore of great importance for predicting the hardness after reheating of the base metal. In this regard, various semiempirical approaches to model the dissolution rate of hardening precipitates in aluminium alloys have been proposed in the literature.

The dissolution model of Myhr and Grong [3] [4] contains a single independent variable, the time, and a single state variable, the volumetric fraction of hardening precipitates. This model provides a basis for obtaining quantitative information of the reaction kinetics through simple hardness measurements, rather than through microscopic observations of the precipitates fraction.

In this regard, Shercliff et al. [5] applied the dissolution model of Myhr and Grong to several aluminium alloys, and applied their results to friction stir welding. They also showed that this does not apply to softer tempers.

Here a neural networks approach is proposed for the identification of metallurgical properties for precipitates dissolution in precipitation hardenable alu- minium alloys. The microstructural modeling of aluminium alloys is formulated as a variational problem including independent parameters.

The novel methodology is here applied to three different aluminium alloys: AA-7449-T79, AA2198-T8 and AA-6005A-T6. The experimental hardness data is taken through independent measurements, and the final software implementation is performed within the Flood library [2].

\section{DISSOLUTION MODEL}

First, assuming that the effect of solid solution hardening is small compared to precipitate hardening at all stages of heat treatment, the following linear relationship between the Vickers hardness and the volumetric fraction of precipitates can be established [3] [4]

$$
\frac{f}{f_{0}}(H V)=\frac{H V-\min (H V)}{\max (H V)-\min (H V)},
$$

where $f / f_{0}$ is the ratio of the current volume fraction of hardening precipitates to the initial volume fraction of hardening precipitates, $H V$ is the actual hardness, $\max (H V)$ is the initial hardness in the hardened state, and $\min (H V)$ is the final hardness after complete dissolution of the precipitates.

On the other hand, the model developed by Myrh and Grong [3] for cylindrical precipitates describes 
the kinetic of transformation of hardening precipitates in aluminium alloys at constant temperature by the following expression,

$$
\frac{f}{f_{0}}(t)=1-\sqrt{\frac{t}{t^{*}(T)}},
$$

where $t$ is the time and $t^{*}(T)$ is the time for complete dissolution at temperature $T$. The full dissolution time $t^{*}(T)$ can be calculated as [3]

$$
t^{*}(T)=t_{R} \exp \left[\frac{Q}{R}\left(\frac{1}{T}-\frac{1}{T_{R}}\right)\right],
$$

where $R$ is the universal gas constant, $t_{R}$ is the reference time for complete dissolution at the reference temperature $T_{R}$ selected for calibration, and $Q$ is the effective activation energy for precipitates dissolution.

It is easy to see that taking $\log \left(1-f / f_{0}\right)$ as a function of $\log \left(t / t^{*}\right)$ in (2), the resulting plot results

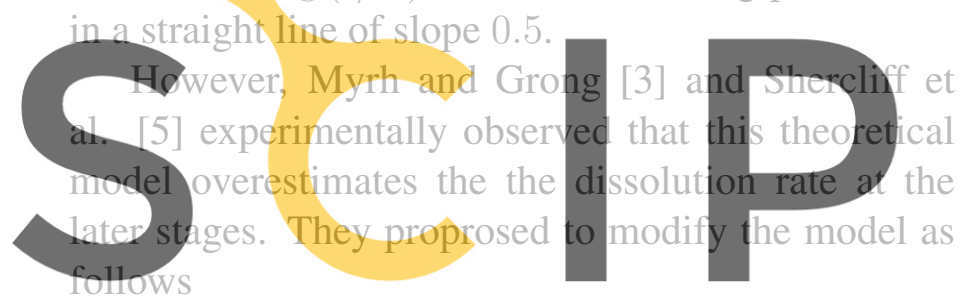

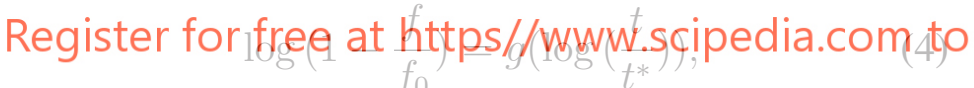

where $g$ is a function whose form is given by a "lookup table". Here the early stages of dissolution follow a straight line of gradient 0.5 , but this slope steadily decreases at the later stages.

The formulation of Myrh and Grong and Shercliff et al. presents some drawbacks. First, the modeling process occurs in two steps, (i) estimation of the effective activation energy and (ii) generation of the dissolution model. This might result in poor accuracy. Second, a "look-up table" is an unsuitable result here, and an explicit expression for the dissolution model is a desirable aim. Third, the logarithmic representation in the $y$-axis produces spurious effects. Indeed, separates the data at the lower stages of dissolution and joins them at the later stages.

The representation of the dissolution model proposed here is of the form

$$
1-\frac{f}{f_{0}}=g\left(\log \left(\frac{t}{t^{*}}\right)\right)
$$

where the function $g$ is to be found from a suitable function space.
The dissolution modeling process consists in estimating an activation energy $Q$ which provides minimum dispersion for the experimental data, while finding a function $g(\cdot)$ which provides minimum error. Mathematically, this can be formulated as a variational problem including independent parameters.

\section{EXPERIMENTAL DATA}

Experimental tests are performed to get the isothermal time evolution of Vickers hardness at different temperatures and for various aluminium alloys. Three materials are used here for the isothermal heat treatments, AA-7449-T79, AA-2198-T8 and AA-6005AT6.

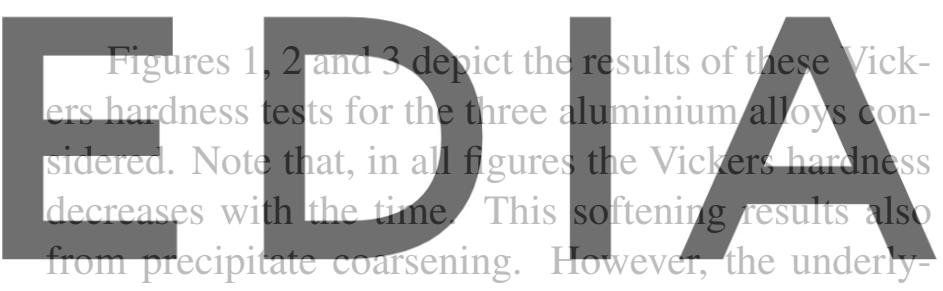

ing mechanism is also controlled by precipitate disdouvalorad the versigm, without the watermark both.

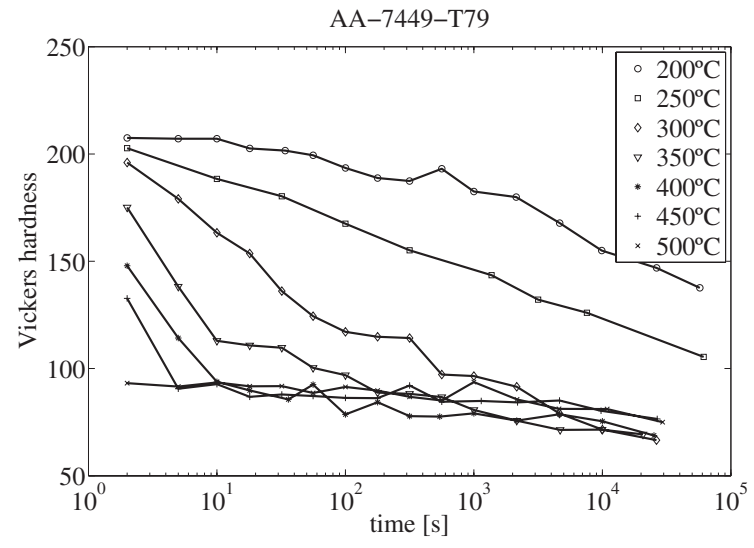

Figure 1: Vickers hardness test for AA-7449-T79. 


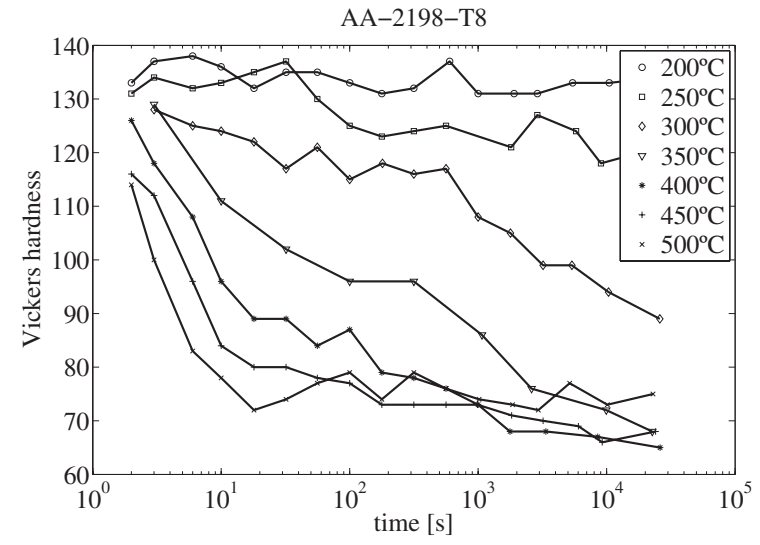

Figure 2: Vickers hardness test for AA-2198-T8.

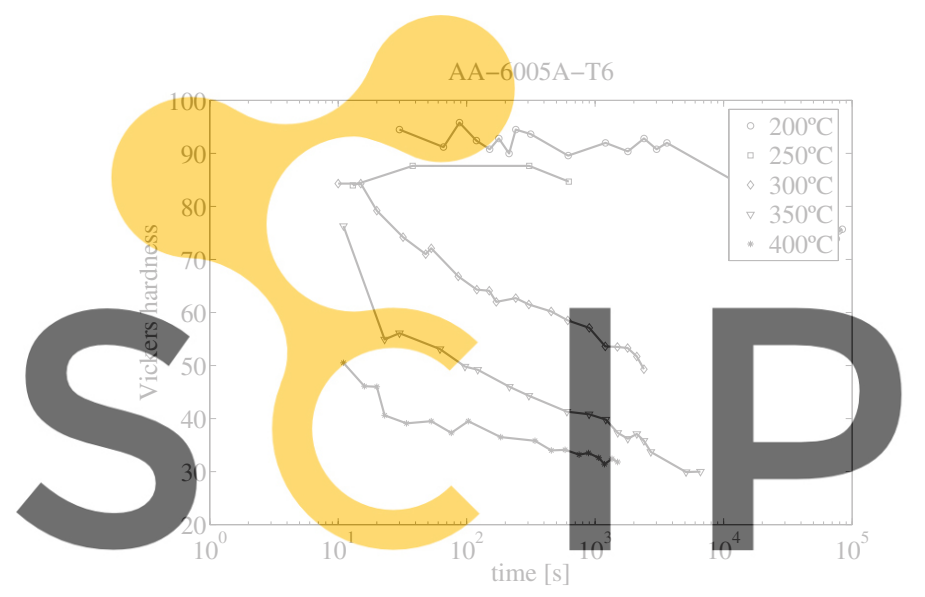

Register for free at https//www. scipedia.com to
Figure 3: Vickers hardness test for AA-6005A-T6.

Table 1 shows the parameters which are needed to transform from Vickers hardness to volumetric fraction of hardening precipitates in (1). Here $m$ and $M$ are the minimum and maximum Vickers hardness; $t_{R}$ and $T_{R}$ are the reference time in seconds and the reference temperature in Celsius.

\begin{tabular}{ccccc}
\hline & $m$ & $M$ & $t_{R}$ & $T_{R}$ \\
\hline AA-7449-T79 & 66.6 & 207.5 & 10000 & 300 \\
AA-2198-T8 & 65 & 138 & 10000 & 350 \\
AA-6005A-T6 & 29.9 & 95.8 & 1000 & 350 \\
\hline
\end{tabular}

Table 1: Parameters for aluminium alloys AA-7449T79, AA-2198-T8 and AA-6005A-T6.

\section{NUMERICAL RESULTS}

A variational formulation for neural networks is in this section applied to find the optimal dissolution model and the effective activation energy of aluminium alloys AA-7449-T79, AA-2198-T8 and AA6005A-T6.

The first step is to choose a function space to represent the dissolution model $\left(1-f / f_{0}\right)\left(\log \left(t / t^{*}\right)\right)$ [2]. Here a multilayer perceptron with a sigmoid hidden layer and a linear output layer is used. This neural network is very useful in inverse problems, since it is a class of universal approximator [1].

Here the multilayer perceptron must have one input, $\log \left(t / t^{*}\right)$, and one output neuron, $1-f / f_{0}$. It is found that the optimal network architecture for the three case studied considered here is that with 1 hidden neuron. On the other hand, information about the effective activation energy is required. Thus, an independent parameter $Q$ must be associated to the multilayer perceptron. This neural network spans a family $V$ of functions $\left(1-f / f_{0}\right)\left(\log \left(t / t^{*} ; \underline{\alpha}, Q\right)\right.$, which are parameterized by the biases and synaptic weights vector $\alpha$ and the effective activation energy $Q$ and has

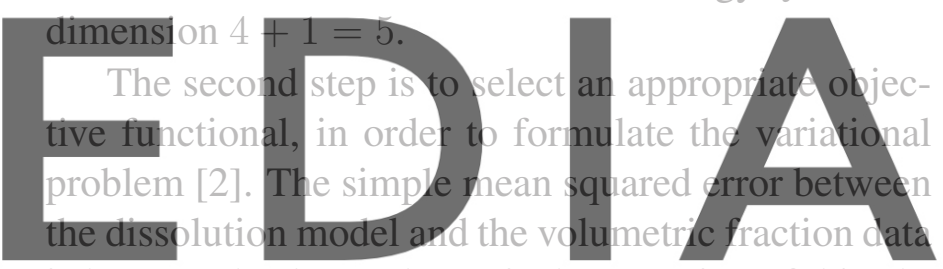

is here used. The mathematical expression of this ob-

$$
F[y(x ; \underline{\alpha}, Q)]=\frac{1}{S} \sum_{s=1}^{S}\left(y(x ; \underline{\alpha}, Q)^{(s)}-y^{(s)}\right)^{2},
$$

where $S$ is the number of samples available, and $y(x ; \underline{\alpha}, Q)^{(s)}$ is calculated by means of (1).

Note that the pairs of values $(x, y)$ in (6) are not fixed, since they depend on the particular value used for the effective activation energy $Q$. Moreover, the optimal value for this independent parameter will provide the minimum dispersion of the data.

The variational problem can then be formulated so as to find a function $y\left(x ; \underline{\alpha}^{*}, Q^{*}\right)$ and an independent parameter $Q^{*}$ for which the objective functional in (6) takes on a minimum value.

The third step is to choose a suitable training algorithm to solve the reduced function optimization problem [2]. To check for presence of local minima a quasi-Newton method with BFGS train direction and Brent optimal train rate methods [2] has been used from different initial guesses. The results provided are in all cases the same, and no local minima seem to appear for these particular case studies. 
Equations (7),(8) and (9) are an explicit expression for the dissolution model of AA-7449-T79, AA2198-T8 and AA-6005A-T6, respectively. Here $x$ denotes $\log t / t^{*}$ and $y$ denotes $1-f / f_{0}$.

$$
\begin{gathered}
y^{*}\left(x ; \alpha^{*}, 140 k J / m o l\right)=0.5[-0.075+0.888 \\
\times \tanh \{1.429+3.671((x+6) / 6-1)\}+1], \\
y^{*}\left(x ; \alpha^{*}, 159 k J / m o l\right)=0.5[0.015+0.870 \\
\times \tanh \{1.037+3.535((x+6) / 6-1)\}+1], \\
y^{*}\left(x ; \alpha^{*}, 176 k J / m o l\right)=0.5[0.044-1.015 \\
\times \tanh \{-1.298-2.835((x+6) / 6-1)\}+1] .
\end{gathered}
$$

Figures 4, 5 and 6 show the results provided by the neural networks for aluminium alloys A A-7449-T79. AA-2198-T8 and AA-6005A-T6, respectively. Comparing the shape of these curves to those reported by Myhr and Grong [3] and Shercliff et al. [5], there are similarities and differences. For medium and high stages of dissolution they are very similar. For early stages the neural network fits the experimental data,

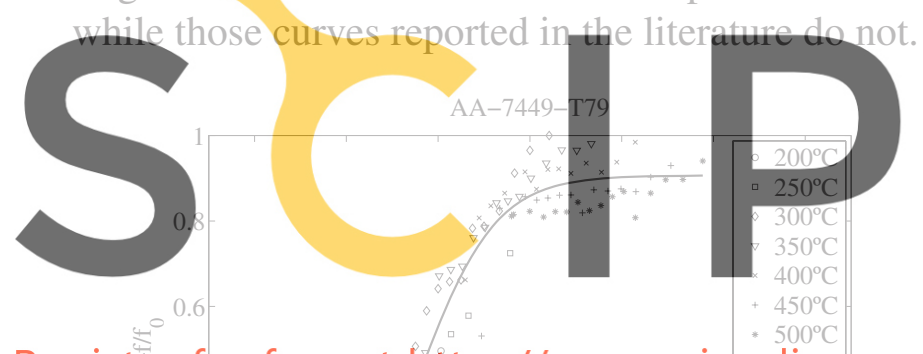

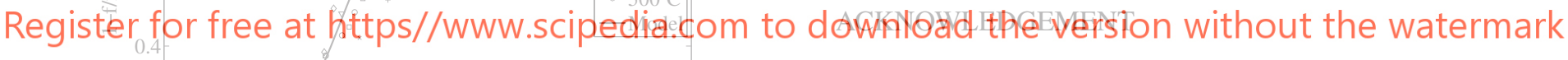

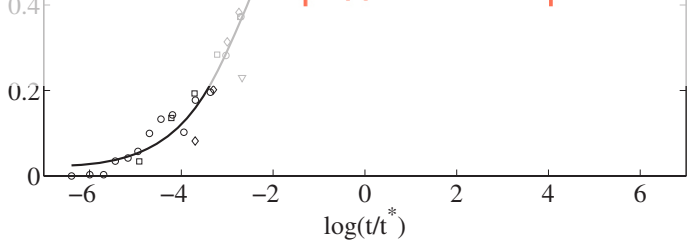

Figure 4: Dissolution model for AA-7449-T79, with $Q^{*}=140 \mathrm{~kJ} / \mathrm{mol}$.

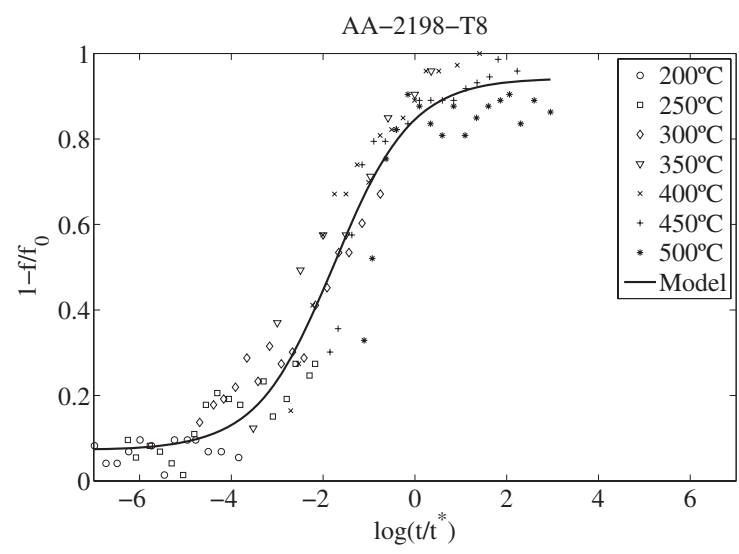

Figure 5: Dissolution model for AA-2198-T8, with $Q^{*}=159 \mathrm{~kJ} / \mathrm{mol}$.

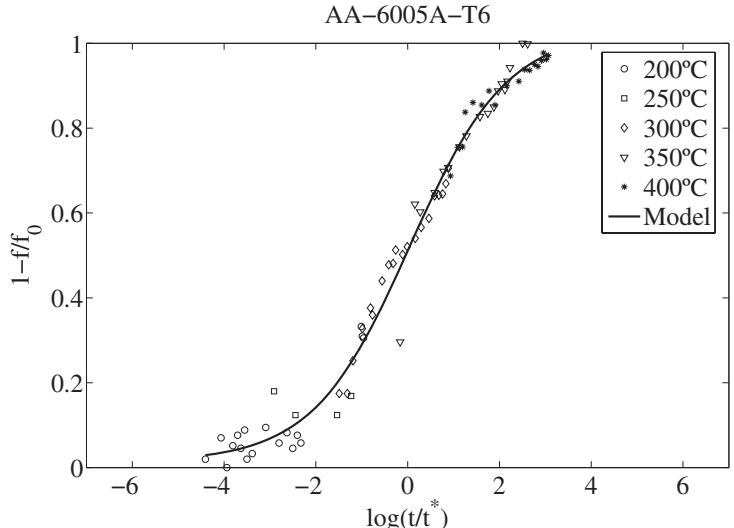

Figure 6: Dissolution model for AA-6005A-T6, with $Q^{*}=176 \mathrm{~kJ} / \mathrm{mol}$.

\section{CONCLUSIONS}

Neural networks were successfully used to find the effective activation energy and model the dissolution

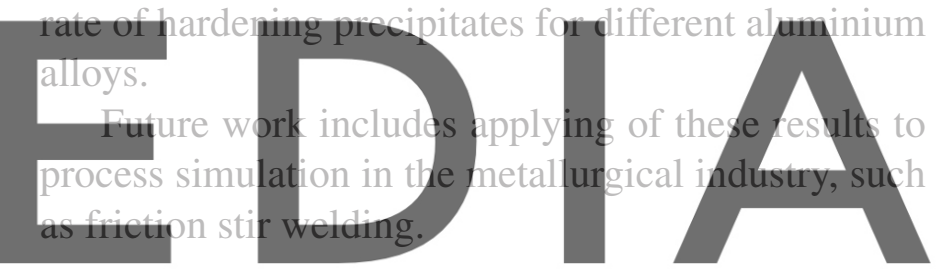

This work has been supported under the 6th Framework Programme of the European Comission through the project "Detailed Multi-Physics Modelling of Friction Stir Welding (DEEPWELD)", Ref. AST4-CT-2005-516134.

\section{REFERENCES}

[1] K. Hornik, M. Stinchcombe, and H. White. Multilayer feedforward networks are universal approximators. Neural Networks, 2(5):359-366, 1989.

[2] R. Lopez. Flood: An open source neural networks c++ library. Www.cimne.com/flood, 2005.

[3] O.R. Myrh and $\varnothing$. Grong. Process modelling applied to 6082-t6 aluminium weldments - i. reaction kinetics. Acta Metallurgica et Materialia, 39(11):2693-2702, 1991.

[4] O.R. Myrh and $\varnothing$. Grong. Process modelling applied to 6082-t6 aluminium weldments - ii. applications of model. Acta Metallurgica et Materialia, 39(11):2703-2708, 1991.

[5] H.R. Shercliff, M.J. Russel, A. Taylor, and T.L. Dickerson. Microstructural modelling in friction stir welding of 2000 series aluminium alloys. Mecanique \& Industries, 6:25-35, 2005. 